\title{
Using surface electromyography to predict single finger forces
}

\author{
Claudio Castellini and Risto Kõiva
}

\begin{abstract}
Surface electromyography (SEMG) of the forearm is an active research topic since the 1990s in the rehabilitation robotics / machine learning community, as it can be used to predict the hand posture and overall grip force. We hereby advance the state of the art by describing a multi-subject experiment in which sEMG is successfully used to predict simultaneous forces applied by a human subject at the fingertips, that is, when six voluntary muscle contractions (VMCs) are elicited (flexion of the little, ring, middle and index fingers, thumb rotation and thumb adduction). Using a multi-sensor setup sEMG activity of the forearm of a human subject and the forces exerted at the fingertips are measured; a Support Vector Machine is then used to associate sEMG signals and forces. Our results clearly show that SEMG can be used to predict the required forces with an error as small as $1.5 \%$ of the sensor range. Targeted positioning of the electrodes is not required. The prediction is uniformly accurate across all VMCs and all 12 subjects considered, and it is robust against subsampling. This result goes in the direction of enabling natural force / impedance control of a highly dexterous prosthetic hand over a continuous, infinite manifold of force configurations, rather than using posture classification like in the traditional approach.
\end{abstract}

\section{INTRODUCTION}

Surface electromyography (sEMG) is a non-invasive technique that measures muscle activation potentials, in use since the 1960s [1] to control self-powered hand prostheses such as, e.g., Otto Bock's SensorHand Speed (www.ottobock.com). The main advantages of this technique are that it requires no surgical intervention and is relatively cheap, although interpreting the signal is difficult [2], [3]. Traditionally, after an amputation is performed and the wounds are healed, a custom socket is designed and built to be placed at the location of the amputee's high-activity forearm muscle remnants, allowing optimal stable placement of two sEMG electrodes over the signal sources. The patient is then trained to contract one or more muscles in order to open or close the prosthesis. (A detailed survey of sEMG and its applications can be found in [4], [5].)

Over the past 20 years however, dexterous mechanical hands have appeared which could enable an amputee move single fingers, if only a sensible control system were available. To this end the scientific community has answered by

This work is supported by the DFG Center of Excellence EXC 277 Cognitive Interaction Technology (CITEC), and by the Swiss National Science Foundation project nr. 132700, NINAPRO (Non-Invasive Adaptive Hand Prosthetics).

C. Castellini (corresponding author) is with the Institute of Robotics and Mechatronics, DLR (German Aerospace Center), D-82234 Weßling, Germany. claudio.castelliniedlr.de

R. Kõiva is with the Neuroinformatics Group, Center of Excellence Cognitive Interaction Technology, Bielefeld University, D-33615 Bielefeld, Germany. risto.koiva@uni-bielefeld.de

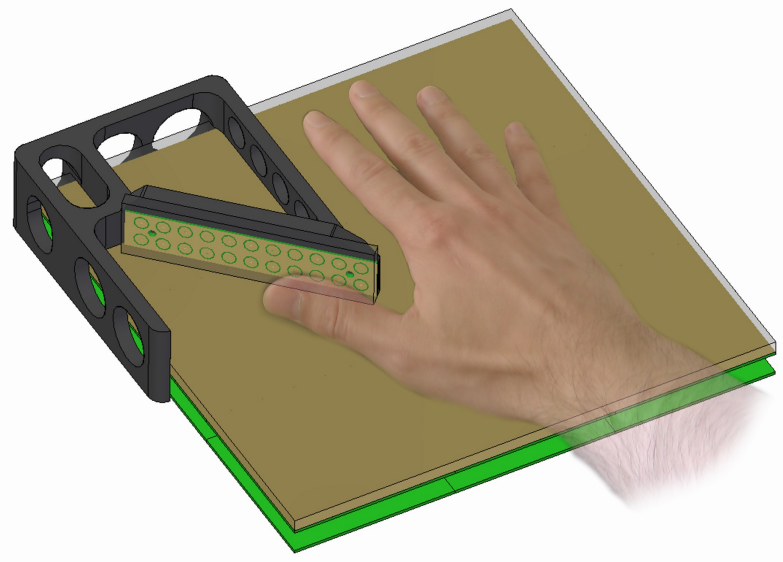

Fig. 1. The Myrmex tactile sensor system and the single iObject tactile module as arranged in our setup. The Myrmex detects the force applied orthogonally to its surface, and is therefore used for the finger flexions (including thumb rotation); the iObject module, mounted orthogonally to the Myrmex surface, detects the thumb adduction. For the sake of clarity, the conductive sensor material of both tactile sensors is displayed translucently.

augmenting the number of electrodes (up to 32 [6]) and by adopting sophisticated statistical / machine-learning methods to decode the signal, that is, to associate it with a desired hand posture and sometimes to the required grasp force. These methods range from Linear Discriminant Analysis [7] and Neural Networks [8] to Support Vector Machines [9]. The approach has also been successfully applied to amputees [10], [6], [11], [12].

So far, however, few attempts have been made at simultaneously predicting the force required by a subject over voluntary muscle contractions (VMCs); for instance, it would be interesting to predict the required force when, e.g., flexing a single finger, or pronating the wrist, etc.; and such a prediction should work also when the finger is flexed while the wrist is pronated. In particular (to the best of our knowledge) no such attempt has been made at all in trying to independently and simultaneously assess finger flexion forces, either alone or when combined. The benefit of such advancement, we claim, would be clear in terms of the activities of daily living that a patient would be able to perform. This is in stark contrast with the traditional approach, mainly based upon static hand posture classification. Classification restricts the user's control of prosthesis to a finite (usually small) number of predefined grip patterns, whereas simultaneous prediction of VMC force would enable selection over an infinite manifold of force configurations. Moreover, such control would be more natural and driven by 
the patient's will, which would greatly improve the prosthesis usability and acceptance [12], [13].

In this paper we present an experiment whose results show that such a control is indeed possible. Twelve ablebodied human subjects have performed repetitive series of VMCs of the right hand, namely, flexion of the little, index, middle and ring fingers, rotation and adduction of the thumb, while nine sEMG electrodes recorded their forearm muscular activity. The electrodes were placed uniformly just below the elbow, without searching for relevant muscles. An adapted tactile sensor unit (Fig. 1) was used to obtain a faithful representation of the force exerted at the fingertips and laterally by the thumb. Support Vector Machines (SVMs) were employed to construct a mapping from the sEMG signals to the detected forces.

Our results indicate that the forces at the fingertips can be accurately predicted from the sEMG signal sampled at $50 \mathrm{~Hz}$. The best prediction error is about $1.5 \%$ of the signal range. The prediction accuracy is uniform across all VMCs considered (even when they happen simultaneously) and across all subjects considered; and it is robust against subsampling, i.e., it degrades reasonably as fewer and fewer samples are used to train the SVMs - this becomes necessary in an online setting or as computational resources are limited.

After a review of related work, we hereafter describe the setup and the experiment, then the methods used for the analysis and the experimental results. Lastly we draw some conclusions and outline future work.

\section{A. Related work}

Literature about sEMG for upper-limb prostheses is nowadays large; the interested reader should refer to, e.g., [4], [5], [13], [14], where broad overviews are given from multiple points of view. The community has mainly focused so far on classification of sEMG signals, meaning that isotonic muscular configurations (i.e., static hand postures) are associated to categories and then predicted as such. For example, in [6], [12] single finger motions are recognized as such by a classifier, meaning that the system is able to tell one finger motion apart from the other, but there is no quantitative hint about the position (for example the angle between hand joints) and/or the force exerted. A more structured result appears in [15], where prediction of finger positions is obtained with more than $90 \%$ correlation to the recorded values of the joint angles.

Here we rather focus upon prediction of force applied at single fingertips (a regression problem rather than a classification). Regression of force for single VMCs has been realized, as far as we know, only in [16], where simultaneous regression upon force exerted by the three degrees of freedom of the human wrist is enforced using an artificial neural network. In general, predicting forces is in our opinion more interesting than predicting positions, for at least two reasons: (1) position control is effective only in the absence of obstacles, which is seldom the case when a hand is engaged in grasping and/or manipulation; and (2) sEMG is well-known to be related to force rather than to position, so regression on positions from sEMG really is regression on isotonic muscular configurations. This is corroborated even by recent results on sEMG-based direct position control of robotic arms [17], [18].

\section{EXPERIMENTAL SETUP AND DESIGN}

The setup consists of three main components: the sEMG electrodes, the tactile sensor and the stimulus visualization.

\section{A. Surface electromyography}

Muscular activity is gathered using 9 OttoBock MyoBock 13E200 surface EMG electrodes. The electrodes provide an amplified, bandpass-filtered and rectified signal, eliminating the need of further external signal conditioning. The electrodes, tied to a hook-and-loop strap using elastic bands, are attached on the subject's forearm, just below the elbow. Five electrodes lay on the dorsal side and four on the ventral side of the forearm. This placement is intentionally largely irrespective of the anatomy of the human forearm: namely, no search for relevant muscles was performed before the straps were secured. Uniform placement, irrespective of anatomy, has already been demonstrated effective, even on amputees [11]. Fig. 2 shows the sensor arrangements and the general setup. The signals from the electrodes are gathered by a standard digital acquisition card at a rate of 500 samples per second.

\section{B. Tactile sensors}

Fingertip forces are recorded using a combination of two tactile sensor systems in a custom setup, arranged optimally for capturing data from a medium-sized adult right hand. The forces generated by flexion of the index, middle, ring and little finger, as well as the rotation (flexion) of the thumb are recorded by an array of Myrmex sensor modules [19]. A single Myrmex sensor module measuring $80 \times 80 \mathrm{~mm}$ includes $16 \times 16$ tactile cells (tactels) and has a spatial resolution of $5 \mathrm{~mm}$. Connecting $3 \times 3$ Myrmex sensor modules, a flat sensor measuring $240 \times 240 \mathrm{~mm}$ was used, resulting in a surface with 2304 tactels. The silhouette of a right hand is taped on top of the sensor in order to give the participant a clear idea of where the hand should be positioned over the sensor surface. (Data from tactels not relevant for the five forces of interest were discarded.) All in all, a variable number of tactels between 28 to 35 were used for each VMC.

On the other hand, thumb adduction force was measured using a tactile sensor derived from the iObject [20]. The iObject is an instrumented object that has the size and shape of a 330ml soft drink can, and was developed for human and robotic manual interaction research. It includes, among other sensor modalities, 10 tactile sensor modules forming its decagon outer shell. In the present setup one of the iObject's tactile sensor modules is mounted vertically on top of the Myrmex modules (see Fig. 1 for a detailed view) to allow measurement of the thumb adduction. The single iObject tactile sensor module has 22 tactels and measures $20 \times 115 \mathrm{~mm}$ achieving a spatial resolution of $10 \mathrm{~mm}$. In this case too, non-relevant sensors were removed from the data 

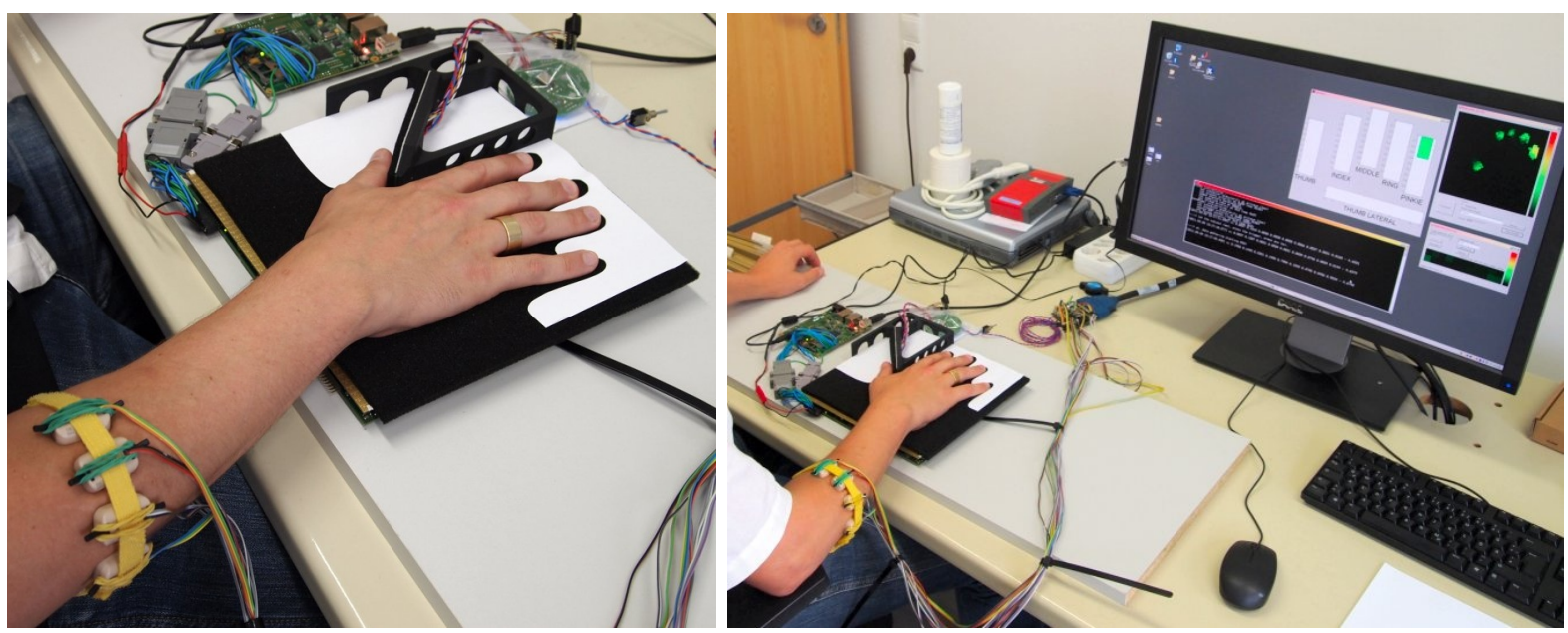

Fig. 2. The experimental setup while in use by a subject (detail on the left). The subject wears 9 sEMG electrodes on the right forearm, lays his right hand on the tactile sensors and is shown the stimuli on the screen. The output of the tactile sensors is displayed in a graphically clear way next to the stimuli to help the subject match the required contraction.

gathering, resulting in 10 significant tactels captured for the thumb adduction.

Both tactile sensors implement a similar resistive sensing working principle giving a characteristic hyperbolic output [21] and allow a fine-grained insight into subtle hand movements, while still being able to output discriminating values for high finger forces (being sensitive in the range from 1 to $30 \mathrm{kPa}$ ). The internal analog-digital-converters of both sensors work with 12-bit resolution and output data at approximately 250 frames-per-second each. Both tactile sensor systems are connected to a PC via USB 2.0 connection.

The tactile sensors were calibrated using a 3-axis numeric control table with an attached strain gauge sensor, sampling the tactels at numerous positions while exerting forces up to $20 \mathrm{~N}$. The raw output of tactels varies slightly due to resistivity inhomogeneity in the used sensor foam and also by the contact position relative to single tactels. This effect is fortunately minimized significantly when considering the summed output of active tactels (as fingers of adults are relatively larger than used tactels in the sensors, multiple tactels always get agitated by single fingers).

\section{Experimental protocol}

Twelve subjects (all right-handed; 9 male, 3 female; age $31.8 \pm 6.9 \mathrm{yrs}$, min. 21, max. 44) joined the experiment. Each participant sat on an office chair which was adjusted to give maximum comfort; the subject's right elbow lay on the chair's armrest and the right hand lay over the tactile sensor. Desired finger positions were given by the hand silhouette fixed over the sensor surface. The armrest of the chair and the Myrmex sensor system were at the same level and height in respect to each other. A monitor was placed at a comfortable distance, on which the stimulus would be shown.

The stimulus consisted of a set of colored bars, the height of the bars and the color simultaneously denoting the targeted VMC of each finger; the colors would match those of the graphical representation of tactile sensor outputs, also displayed on the same monitor. During the experiment the subject was asked to match the forces, as faithfully as possible, using the visual stimulus and visual feedback of the tactile sensors as guidance. (The movie "sEMGprediction.mp4", provided as supplemental material, shows two example sequences of the experiment.)

The application showing the stimulus was controlled at $25 \mathrm{~Hz}$ on the same PC, and the displayed force magnitudes were saved on disk for later offline processing. Before the beginning of the experiment, a short training phase happened, so that the participant would familiarize with the setup and the required task. For instance, a bar labeled "RING" would appear, denoting the required amount of contraction; the bar would move from 0 (minimum, in dark green) to 1 (maximum, in red) while the subject would try and apply such force at the tip of the ring finger, in order to match the output color of the tactile sensors graphical output with the colored bar.

The VMCs provided as stimulus were single-finger contractions (little, ring, middle, index and thumb full flexion and return to idle, and thumb full adduction with return to idle) and multi-finger contractions (simultaneous flexion of ring+middle, little+ring+middle, little+ring+middle+index, little+ring+middle+thumb.rotation and little+ring+middle+thumb.adduction). Each contraction was performed in 6 seconds, with 2 seconds of rest in between, and repeated sequentially 5 times. All in all 60 contractions were performed, for a total duration of approximately 7 minutes. No fatigue or discomfort was reported by any of the subjects.

\section{DATA ANALYSIS}

\section{A. Synchronization, preprocessing, consistency}

Data synchronization is enforced on a Windows PC equipped with a multi-core processor, by gathering data from each device asynchronously and accurately timestamping each received datum. Timestamping is enforced by the HRT 

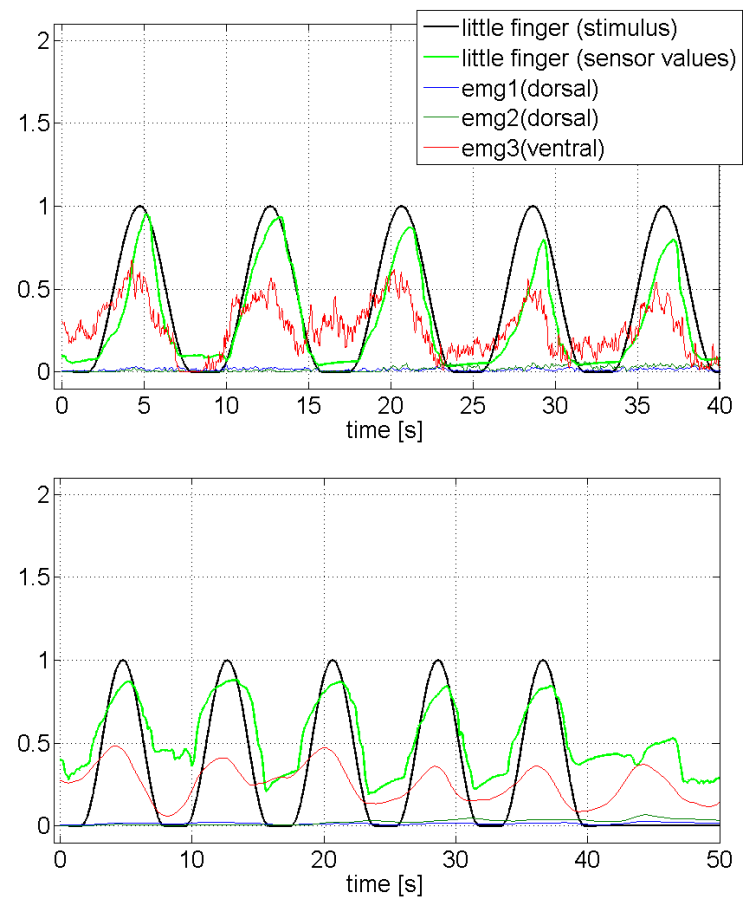
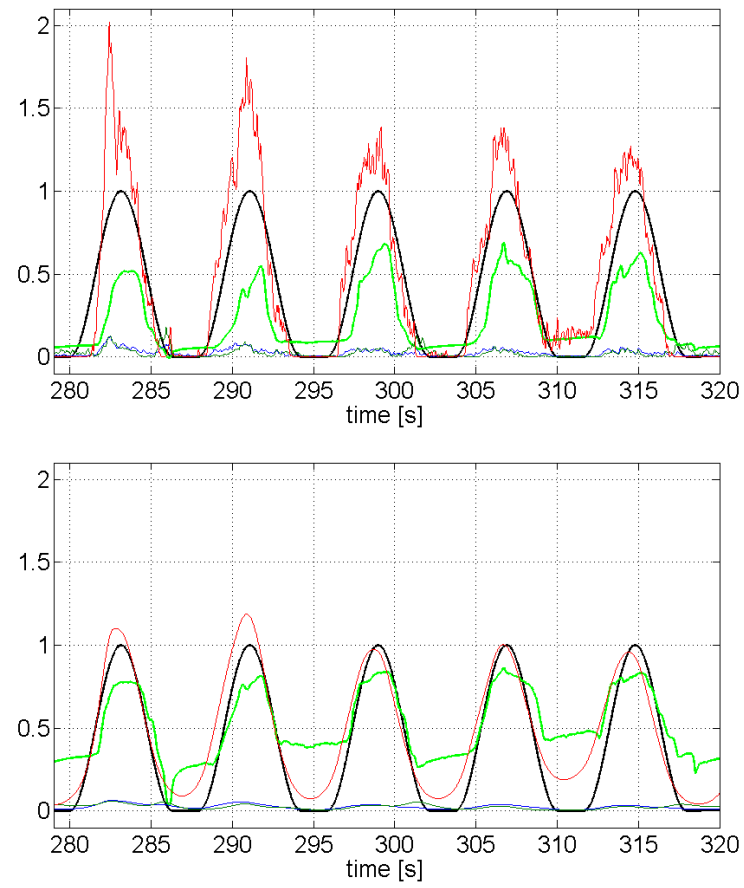

Fig. 3. Typical data gathered during the experiment. (top left) flexion of the little finger, (top right) flexion of little+ring+middle; (bottom row) same data as the top row but with a $0.2 \mathrm{~Hz}$ cutoff lowpass filter on the sEMG signals. See also the movie "sEMGprediction.mp4", provided as supplemental material.

library [22], giving a precision of up to $1.9 \mu \mathrm{s}$. Linear interpolation is used to find the tactile and stimulus values best corresponding to the time at which each sEMG sample is received on the PC. All data are therefore supersampled at $500 \mathrm{~Hz}$ (data from the tactile sensors were originally sampled at about $250 \mathrm{~Hz}$, while the stimulus produced data at a rate of $25 \mathrm{~Hz}$ ), resulting in about $215 \mathrm{~K}$ samples per each data stream. Using a hardware trigger connected to the tactile sensors and to the digital acquisition card, the iObject tactile module was found, in an early round of timing experiments, to bear a systematic delay of $200 \mathrm{~ms}$ on average. This delay has been compensated during the data synchronization. Furthermore, the curves obtained during the calibration phases of the tactile sensors (see the previous Section) have been used to correct the raw values and obtain a quasi-linear response. The resulting force values have then been normalized in the interval 0-1 (no force / maximum force applied).

Data consistency has been ensured thanks to an extensive visual analysis of the obtained signals. Consider Fig. 3, top row, showing the typical stimulus signal of the little finger (i.e., what was shown as guidance for the subject's little finger flexion), the actual tactile sensor values for the little finger, and three prominent sEMG electrodes. The left panel shows the signals while only the little finger flexion was stimulated: the tactile values follow closely the stimulus, while the ventral electrode shows a strong, coordinated response (little or no response is found at the dorsal electrodes). This is consistent with the fact that flexing the little finger only involves almost exclusively the flexor muscles. On the other hand, the right panel shows the same signals, but this time flexion of the little+ring+middle is stimulated: the little- finger stimulus is still there, the tactile response is weaker (since now the force is applied by the three fingers altogether) and the sEMG response is increased; in particular, the dorsal electrodes are responding, too, as a sign of increased muscle co-contraction.

All data have then been low-pass filtered using a $1^{\text {st }}$ order digital Butterworth filter with cutoff frequency at $5 \mathrm{~Hz}$, except for the sEMG data, which has been filtered with the same filter but with lower cutoff frequencies, namely between $1 \mathrm{~Hz}$ and $0.2 \mathrm{~Hz}$. We took care that no unacceptable delays and/or offsets were introduced by this step: delays are avoided by using double-sided filtering (this is unproblematic in an offline setting such as this), while offsets were checked visually (see Figure 3, bottom panel).

\section{B. Analysis method}

The focus of this paper lies in checking the feasibility of the approach, so we used a standard supervised learning method to build a map from sEMG to finger forces, namely Support Vector Machines (SVM, [23], [24]), a technique which has already been proved effective in literature (e.g., [8]). In this case the input space is the space of sEMG signals, $\mathbb{R}^{9}$, whereas the output space is the set of force values for each VMC considered, that is $\mathbb{R}^{6}$. For each experiment, a certain subset of the whole dataset is used for training (training set) and what is left is used for testing; training samples are normalized by subtracting the mean values and dividing by the standard deviations, dimensionwise; testing samples are normalized analogously but using the statistics evaluated on the training set. Since standard SVMs have no support for multi-variate outputs, 6 SVMs 
were trained independently, one for each VMC considered. Grid-search was used in an initial round of experiments to find out the optimal values of the hyperparameters $\sigma$ and $C$ (a constant involved in the cost function minimization problem), revealing especially that the best results are obtained with a value of $C$ around 100. For each experiment, 10-fold crossvalidation is used as follows: ten different random permutations of the data set are generated, and then the SVMs are trained each time on a subset of the data set and tested on the remaining samples. The resulting 10 error values are then presented as mean plus/minus one standard deviation. The chosen error metric is the Root-Mean-Squared-Error normalized over the range of the target values in the testing set (NRMSE). In the case of multi-subject analysis, the 10 error values (one per data permutation) are averaged out for each subject and the resulting 12 error values (this time, one per subject) are presented as mean plus/minus one standard error of the mean.

\section{EXPERIMENT RESULTS}

\section{A. Single-subject analysis}

The regression error has been analyzed for filter cutoff frequencies of $1,0.5,0.3,0.2 \mathrm{~Hz}$, and as fewer and fewer samples are used to train the related SVMs, initially for a typical subject. In particular, we evaluated the NRMSE for training sets with $\frac{1}{k}$ samples, for $k=10,15,20,25,30,35,40,45,50$. In practice, as $k$ is raised, one tenth, one fifteenth, ..., one fiftieth of the total data set is used to train the SVMs. Fig. 4 shows the obtained results. As it was expected, as fewer and fewer samples are used for training, the NRMSE grows monotonically and seemingly less than linearly. The best results are obtained while predicting little flexion, ring flexion and thumb adduction, while index flexion and thumb rotation are the worst; this is somehow surprising for the index flexion, while it is expected for the thumb rotation, since the muscle activity related to this VMC is mainly confined near the wrist, that is, far away from the electrodes. Standard deviations are uniformly rather low if compared with the related means. Setting a lower cutoff frequency for the sEMG lowpass filter sensibly improves the accuracy. The best result is obtained on the little flexion for $k=10$ and cutoff at $0.2 \mathrm{~Hz}$, where the NRMSE is $2.05 \% \pm 0.08 \%$. Fig. 5 shows two typical cases of recorded and predicted force values.

\section{B. Multi-subject analysis}

In order to check that the single-subject result would hold in the general case, we picked up three typical filter/subsampling settings and repeated the analysis on the data gathered from all subjects. Namely, we chose a cutoff frequency of $0.2 \mathrm{~Hz}$ and $k=10,30,50$. Fig. 6 (top panel) shows the results.

As one can see, multi-subject results confirm the singlesubject results: the best accuracy overall ranges from $1.53 \% \pm 0.14 \%$ ( $k=10$, thumb adduction) to $7.67 \% \pm 0.58 \%$ ( $k=50$, thumb rotation). As $k$ is increased the accuracy uniformly degrades; thumb adduction is the easiest VMC to

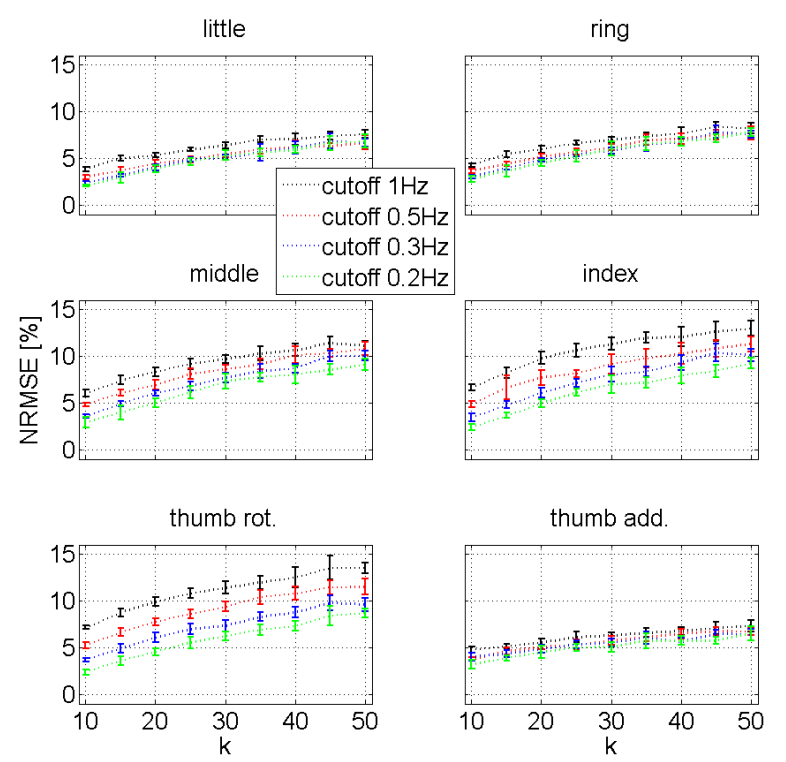

Fig. 4. Single-subject analysis. Prediction error for each VMC, with $1 \mathrm{~Hz}$, $0.5 \mathrm{~Hz}, 0.3 \mathrm{~Hz}$ and $0.2 \mathrm{~Hz}$ lowpass filter cutoff frequencies, and as fewer and fewer samples are used as a training set (namely, $\frac{1}{k}$ of the total dataset is used).

predict, and thumb rotation the hardest. Results are uniform across subjects and robust, the standard errors of the means being uniformly small with respect to the average values. For example, for $k=50$, the error values are $6.65 \% \pm 0.23 \%$, $6.14 \% \pm 0.28 \%, 6.44 \% \pm 0.40 \%, 5.88 \% \pm 0.42 \%, 7.67 \% \pm$ $0.58 \%$ and $4.63 \% \pm 0.27 \%$.

\section{Combining VMCs}

A further interesting result comes from the comparison of the multi-subject error rates obtained on single-VMC data, versus multi-VMC data - in other words, we analyzed whether the activation of many VMCs at the same time would be more problematic than single VMCs. As displayed in Fig. 6 (bottom panel), the prediction of multi-finger VMCs is consistently harder than single-finger ones. Interestingly, the overall multi-subject prediction error (consider the top panel once again) is almost uniformly in-between the singleand multi-finger values. For example, the flexion of the little finger (top panel, first column, for $k=30$ ) has an overall error of $5.06 \% \pm 0.19 \%$; for the same VMC, the singlefinger dataset gives an error of $3.73 \% \pm 0.33 \%$, whereas the multi-finger data yields $6.13 \% \pm 0.24 \%$. Notice however that, overall, even the multi-finger prediction error is always less than $8 \%$.

\section{CONCLUSIONS AND FUTURE WORK}

\section{A. Conclusions and discussion}

Hand/wrist posture classification and force regression is a hot topic in the community since at least 20 years. In this paper we have introduced the (so far informal) concept of voluntary muscle contraction (VMC), and argued that simultaneously predicting the required forces for a set of relevant 

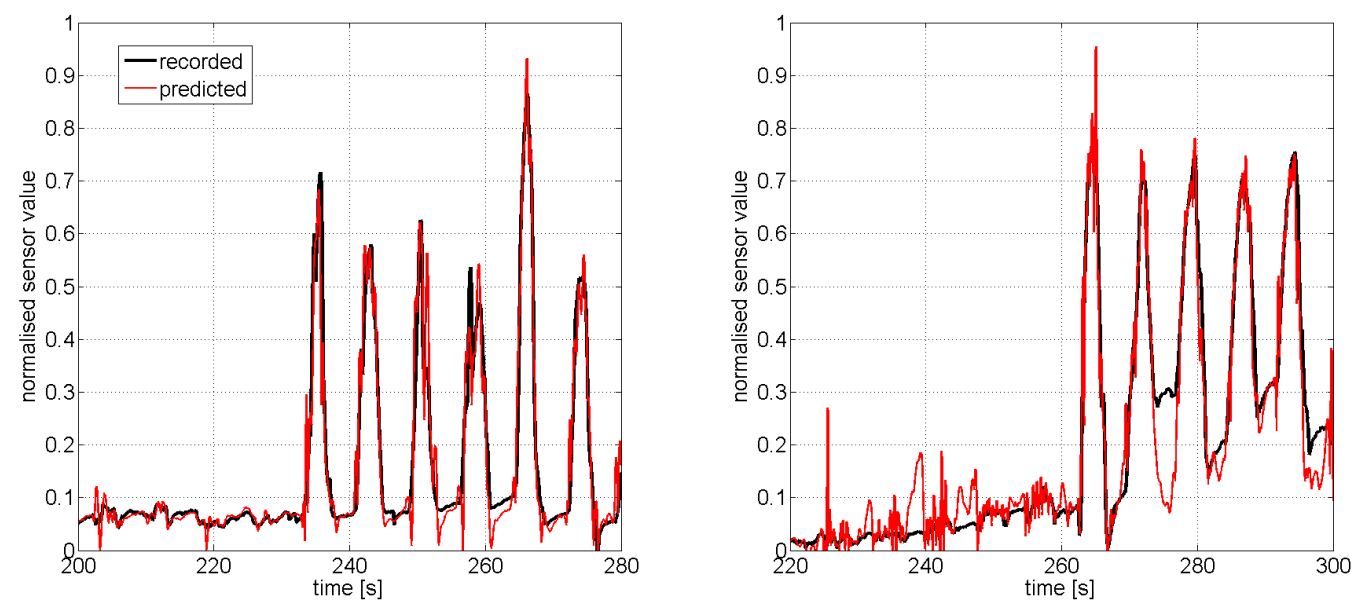

Fig. 5. Recorded and predicted force values for a typical subject. (left) Little finger flexion, cutoff at $0.5 \mathrm{~Hz}, k=20$; (right) thumb rotation, cutoff at $0.5 \mathrm{~Hz}, k=15$.
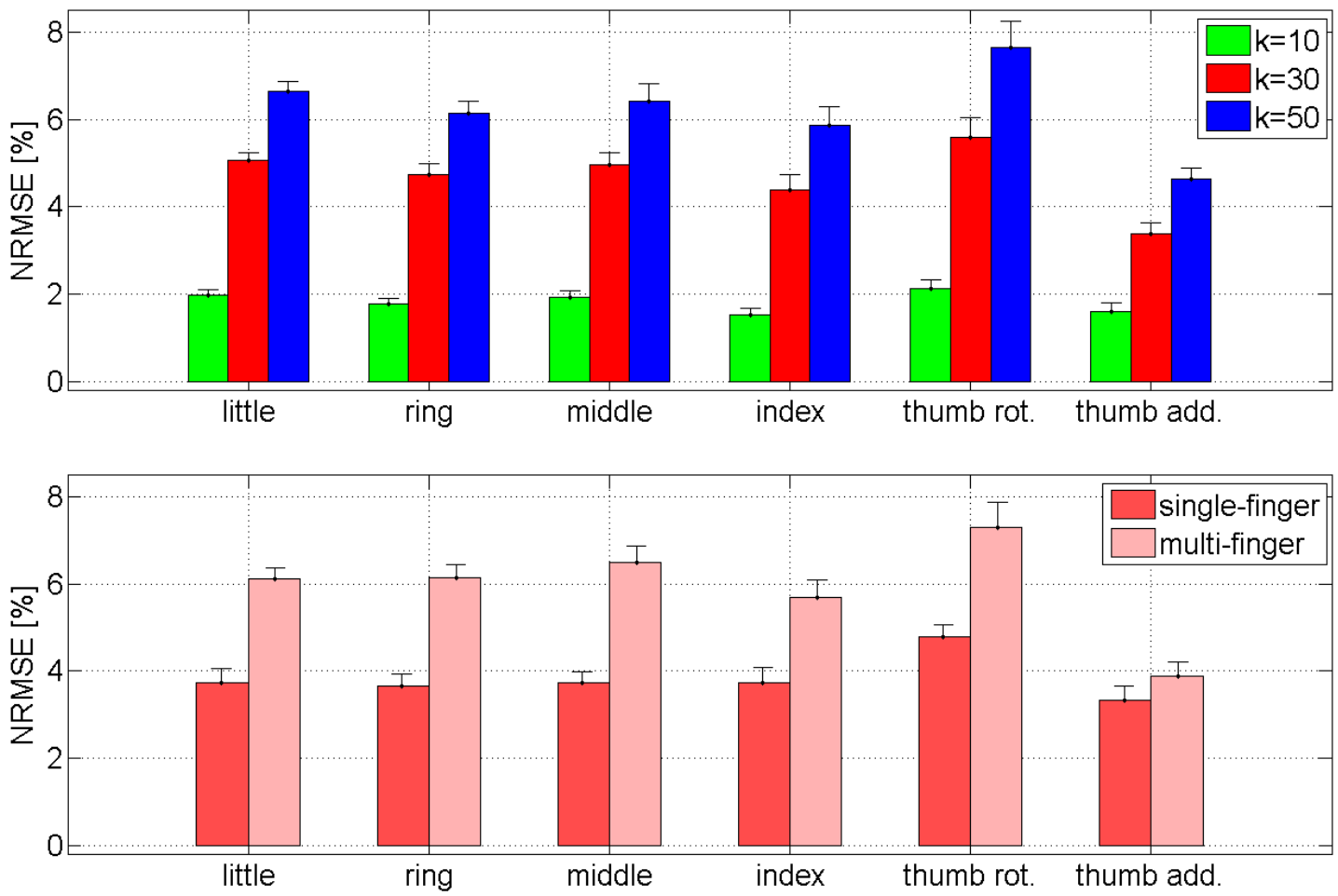

Fig. 6. (top panel) Prediction error for each VMC, for $0.2 \mathrm{~Hz}$ lowpass filter cutoff frequency and $k=10,30,50$. (bottom panel) Prediction error for each VMC, for $0.2 \mathrm{~Hz}$ lowpass filter cutoff frequency and $k=30$, on single- and multi-finger VMCs. (Average values plus/minus one standard error of the mean over 12 subjects.)

VMCs is better than the standard approach. In particular, we argue that this approach enables the human subject to achieve an infinite manifold of force configurations, which is better suited to accomplish activities of daily living especially if the hand prosthesis is impedance-controlled.

The experimental results we have presented clearly show that sEMG can be effectively used to enforce this approach. In an experiment performed on 12 intact subjects, we have chosen six VMCs, namely single and combined flexion of the fingers, thumb rotation and adduction, and predicted the required force up to a precision of about $1.5 \%$ of the target range. The prediction is uniformly accurate across the six VMCs and across the 12 subjects, and it is robust against subsampling. Uniform positioning of the electrodes is used, meaning that no search for target muscles is required.

The fact that regression errors are consistently worse when a dataset of multi-finger VMCs is employed, with respect to the single-finger case, seems to indicate that the 
single-finger dataset is somehow self-contained, or "more clearly separated" than the other. We conjecture that, in the single-finger dataset, training on a particular VMC will enable prediction for that same $\mathrm{VMC}$, without affecting the prediction of the other VMCs. This is probably not the case in the multi-finger dataset, where, e.g., flexion of the ring+middle should be statistically quite similar to flexion of the little+ring+middle. In other words, it might be the case that the sEMG signal can be fruitfully decomposed by choosing a suitable set of simple VMCs and then projecting the more complex ones onto the former ones. We argue that a source-separation technique (e.g., PCA, ICA or BSS) might be useful here.

\section{B. Future Work}

An immediate extension of this work consists of testing the presented approach on amputees, since remarkable residual muscular activity is present in the stump of most trans-radial amputees [10], [6], [9], [12] and training can be enforced via imitation, bilateral coordinated action or the use of mirrors [11]. Whether our approach is feasible in practice is a subject of future research, and a few further points need to be investigated; for instance, how to effectively train the system, as no ground truth is available from amputees (steps in this direction appear in [11], [16]). From the point of view of machine learning, building an online system is an orthogonal, interesting direction of research, analogous to that of [12].

\section{ACKNOWLEDGMENTS}

The authors would like to thank Carsten Schürmann of Bielefeld University for developing the Myrmex tactile sensor modules and Patrick van der Smagt of DLR for actively supporting this project. This work is partially supported by the Swiss National Science Foundation Sinergia project Ninapro (Non-Invasive Adaptive Prosthetics).

\section{REFERENCES}

[1] A. H. Bottomley, "Myoelectric control of powered prostheses," J Bone Joint Surg, vol. B47, pp. 411-415, 1965.

[2] C. J. De Luca, "The use of surface electromyography in biomechanics," Journal of Applied Biomechanics, vol. 13, no. 2, pp. 135-163, 1997.

[3] M. Zecca, S. Micera, M. C. Carrozza, and P. Dario, "Control of multifunctional prosthetic hands by processing the electromyographic signal," Critical Reviews in Biomedical Engineering, vol. 30, no. 4-6, pp. 459-485, 2002.

[4] R. Merletti, M. Aventaggiato, A. Botter, A. Holobar, H. Marateb, and T. Vieira, "Advances in surface EMG: Recent progress in detection and processing techniques," Critical reviews in biomedical engineering, vol. 38, no. 4, pp. 305-345, 2011.

[5] R. Merletti, A. Botter, C. Cescon, M. Minetto, and T. Vieira, "Advances in surface EMG: Recent progress in clinical research applications," Critical reviews in biomedical engineering, vol. 38, no. 4, pp. 347-379, 2011.

[6] F. V. Tenore, A. Ramos, A. Fahmy, S. Acharya, R. Etienne-Cummings, and N. V. Thakor, "Decoding of individuated finger movements using surface electromyography," IEEE Trans. Biomed. Eng., vol. 56, no. 5, pp. 1427-1434, 2009.

[7] M. Leon, J. Gutierrez, L. Leija, and R. Munoz, "EMG pattern recognition using support vector machines classifier for myoelectric control purposes," in 2011 Pan American Health Care Exchanges (PAHCE), april 2011, pp. $175-178$.
[8] S. Bitzer and P. van der Smagt, "Learning EMG control of a robotic hand: Towards active prostheses," in Proceedings of ICRA, International Conference on Robotics and Automation, Orlando, Florida, USA, May 2006, pp. 2819-2823.

[9] C. Castellini and P. van der Smagt, "Surface EMG in advanced hand prosthetics," Biological Cybernetics, vol. 100, no. 1, pp. 35-47, 2009.

[10] F. C. P. Sebelius, B. N. Rosén, and G. N. Lundborg, "Refined myoelectric control in below-elbow amputees using artificial neural networks and a data glove," Journal of Hand Surgery, vol. 30A, no. 4, pp. 780-789, 2005.

[11] C. Castellini, E. Gruppioni, A. Davalli, and G. Sandini, "Fine detection of grasp force and posture by amputees via surface electromyography," Journal of Physiology (Paris), vol. 103, no. 3-5, pp. 255-262, 2009.

[12] C. Cipriani, C. Antfolk, M. Controzzi, G. Lundborg, B. Rosen, M. Carrozza, and F. Sebelius, "Online myoelectric control of a dexterous hand prosthesis by transradial amputees," Neural Systems and Rehabilitation Engineering, IEEE Transactions on, vol. 19, no. 3, pp. 260-270, June 2011.

[13] B. Peerdeman, D. Boere, H. Witteveen, R. Huis in 't Veld, H. Hermens, S. Stramigioli, H. Rietman, P. Veltink, and S. Misra, "Myoelectric forearm prostheses: State of the art from a user-centered perspective," J Rehabil Res Dev., vol. 48, no. 6, pp. 719-738, 2011.

[14] S. Micera, J. Carpaneto, and S. Raspopovic, "Control of hand prostheses using peripheral information," IEEE Reviews in Biomedical Engineering, vol. 3, pp. 48-68, 2010.

[15] R. J. Smith, F. Tenore, D. Huberdeau, R. Etienne-Cummings, and N. V. Thakor, "Continuous decoding of finger position from surface emg signals for the control of powered prostheses," in Engineering in Medicine and Biology Society, 2008. EMBS 2008. 30th Annual International Conference of the IEEE, aug. 2008, pp. $197-200$.

[16] J. Nielsen, S. Holmgaard, N. Jiang, K. Englehart, D. Farina, and P. Parker, "Simultaneous and proportional force estimation for multifunction myoelectric prostheses using mirrored bilateral training," Biomedical Engineering, IEEE Transactions on, vol. 58, no. 3, pp. $681-688$, march 2011.

[17] P. Artemiadis and K. Kyriakopoulos, "EMG-based control of a robot arm using low-dimensional embeddings," Robotics, IEEE Transactions on, vol. 26, no. 2, pp. $393-398$, april 2010.

[18] J. Vogel, C. Castellini, and P. van der Smagt, "EMG-based teleoperation and manipulation with the DLR LWR-III," in Proceedings of IROS - International Conference on Intelligent Robots and Systems, 2011, pp. 672-678.

[19] C. Schürmann, R. Kõiva, and R. Haschke, "A modular high-speed tactile sensor for human manipulation research," in World Haptics Conference (WHC), June 2011.

[20] R. Kõiva, R. Haschke, and H. Ritter, "Development of an intelligent object for grasp and manipulation research," in International Conference on Advanced Robotics (ICAR), June 2011.

[21] K. Weiss and H. Wörn, "The working principle of resistive tactile sensor cells," in IEEE International Conference on Mechatronics and Automation (IEEE ICMA), vol. 1, 2005, pp. 471 - 476.

[22] J. Nilsson, "Implementing a continuously updating, high-resolution time provider for windows," The MSDN Magazine, 2004. [Online]. Available: http://msdn.microsoft.com/en-us/magazine/cc163996.aspx

[23] B. E. Boser, I. M. Guyon, and V. N. Vapnik, "A training algorithm for optimal margin classifiers," in Proceedings of the 5th Annual ACM Workshop on Computational Learning Theory, D. Haussler, Ed. ACM press, 1992, pp. 144-152.

[24] V. N. Vapnik, Statistical Learning Theory. New York: John Wiley and Sons, 1998. 